\title{
Dinámica discreta en un modelo de crecimiento de Solow con ahorro diferencial constante $y$ crecimiento poblacional no constante
}

Fernando Suárez ${ }^{1}$

\section{Resumen}

El presente trabajo considera el modelo de crecimiento de Solow en tiempo discreto, con tasas de ahorro constantes y distintas para los trabajadores y accionistas, y tasa de crecimiento poblacional no constante dada por la ecuación de Richards discreta. Se estudia su comportamiento dinámico incluyendo la aparición de ciclos y caos, complementando herramientas analíticas con simulación computacional. Finalmente se cuantifica la aparición de caos mediante la utilización de exponentes de Lyapunov, interpretando el modelo presentado en términos económicos.

Palabras claves: métodos matemáticos, sistemas dinámicos discretos, mapas bidimensionales no inversibles, crecimiento económico

\section{Abstract}

This paper considers the Solow economic growth model in discrete-time, with constant but different saving rates for workers and shareholders, and non-constant population growth rate given by discrete Richards's equation. Its dynamical behavior is investigated including cycles and chaos emerging, mixing analytical tools, and computational simulations. Finally, chaos emerging is quantified using Lyapunov exponents, understanding the introduced model in economic terms.

Keywords: mathematical methods, discrete dynamical systems, bidimensional noninvertible maps, economic growth.

JEL: C62, C63, 040

DOI: https://doi.org/10.46553/ensayos.3.3.2021.p25-39

Fecha de recepción: 08/04/2021; Fecha de aceptación: 03/08/2021

1 Departamento de Matemática, Facultad de Ingeniería y Ciencias Agrarias, Universidad Católica Argentina, Alicia M. de Justo 1500, (C1107AAZ) C.A.B.A, Argentina. Email: fernandosuarez@uca.edu.ar 


\section{Introducción}

En los últimos años se ha observado un interés creciente por el estudio de sistemas dinámicos no lineales en economía, en la medida en que los modelos económicos a menudo presentaban dinámicas complejas, sobre todo en el campo del crecimiento económico. Este hecho motivó a muchos autores a encarar el comportamiento asintótico de los modelos, sus propiedades y también la eventual aparición de dinámicas caóticas.

Los modelos dinámicos de crecimiento económico han considerado a menudo el modelo estándar neoclásico de Ramsey o el de Solow-Swan (Solow, 1956). Estos sistemas dinámicos convergen al estado estacionario (por ejemplo, el equilibrio del stock de capital per cápita) por lo que no conducen a ciclos ni a dinámicas complejas. Sin embargo, para ser más precisos, debemos aclarar que el modelo de Ramsey supone que el comportamiento del ahorro es tal que corresponde a maximizar la suma de las utilidades descontadas de un consumidor representativo con horizonte infinito, mientras que en el modelo de Solow-Swan se asume que la propensión al ahorro en promedio es constante.

La posibilidad de aparición de dinámicas complejas en modelos de crecimiento óptimo, incluyendo la aparición de caos, ha sido demostrada por Benhabib \& Nishimura (1985), y Boldrin \& Montrucchio (1986) cuando dos sectores se incluyen en dichos modelos.

Posteriormente otros autores consideraron diferentes propensiones al ahorro para dos grupos (trabajo y capital) dando lugar a equilibrios inestables y múltiples, como el caso de Kaldor (1955) y Chiang (1973), entre otros. Si bien con la modificación introducida se logra influir en la dinámica final del sistema, su dinámica cualitativa aún permanece relativamente simple.

El rol de las tasas de ahorro diferenciales pero constantes es recientemente retomado en 2000 por Böhm \& Kaas (2000) en relación con el comportamiento cualitativo del modelo de crecimiento de Solow en tiempo discreto, donde el mismo exhibe inestabilidades y caos. En dicho modelo se vincula el stock de capital por trabajador $k$ con el producto por trabajador $y=f(k)$, siendo $f$ una función de producción genérica que satisface las condiciones de Inada, o sea, es estrictamente creciente, estrictamente cóncava y tal que, $\lim _{k \rightarrow \infty} \frac{f(k)}{k}=0$ y $\lim _{k \rightarrow 0} \frac{f(k)}{k}=\infty$.

En este modelo la fuerza de trabajo crece a tasa constante, hipótesis usualmente asumida en distintos escenarios de crecimiento económico. Sin embargo, una implicancia de considerar la tasa de crecimiento poblacional constante es que la fuerza de trabajo crece exponencialmente, lo cual es claramente no realista. De hecho, una consideración lógica es que siempre existe un límite que es llamado capacidad de acarreo del entorno.

Un modelo de crecimiento más realista debería considerar que cuando la población es pequeña en relación a la capacidad de acarreo del entorno, entonces crece a una tasa constante; en cambio cuando la población es mayor en relación a la capacidad de acarreo 
del entorno, los recursos resultan relativamente más escasos y como resultado esto debería afectar de forma negativa al crecimiento de dicha población.

A partir del modelo empleado por Böhm \& Kaas, Brianzoni et al. (2007a, 2007b) han introducido modelos alternativos teniendo en cuenta la anterior consideración, utilizando distintos mapas para modelizar la fuerza de trabajo.

El presente trabajo considera el modelo de Solow en tiempo discreto, asumiendo la función de producción CES, y tasas de ahorro diferenciales pero constantes. Se introduce el mapa logístico generalizado conocido como ecuación de Richards para modelizar la fuerza de trabajo.

La elección de la ecuación de Richards está justificada porque la misma permite una mejor descripción de la dinámica de crecimiento de la fuerza de trabajo, al disponer de mayor flexibilidad en la ubicación del punto de inflexión de la curva. Al introducir estas funciones en el modelo se manifiestan nuevas posibilidades de equilibrios múltiples, dinámicas complejas y fluctuaciones, no contempladas en los modelos anteriormente descriptos.

Sobre la base de esta elección el objetivo del trabajo es poner en evidencia el rol que juegan los parámetros del mapa de Richards y de la función de producción CES en la posible aparición de estas dinámicas complejas, a saber, ciclos de altos períodos o caos. Los resultados obtenidos apuntan a confirmar el rol central que juega la elasticidad de substitución en la aparición y propagación de las mismas, en relación a la tasa de crecimiento poblacional y al resto de los parámetros del sistema.

El análisis desarrollado brinda las condiciones de estabilidad del modelo, junto con un estudio detallado de los valores de los parámetros que pueden presentar fluctuaciones en la dinámica de crecimiento económico, como aporte complementario a la toma de decisiones de política.

En lo que sigue, este trabajo está organizado de la siguiente manera. En la segunda sección se desarrolla el modelo y se obtiene el sistema dinámico triangular. La tercera sección es un análisis local de la estabilidad de los puntos fijos que pertenecen al sistema. La sección cuarta se dedica al análisis de ciclos y dinámicas complejas, en tanto que la quinta sección estudia mediante simulaciones numéricas el incremento en la complejidad de la dinámica asintótica del sistema utilizando diagramas de fase. La sexta sección introduce una forma alternativa de poner en evidencia la presencia de caos, cuantificándolo mediante exponentes de Lyapunov. La séptima sección recoge las interpretaciones económicas del modelo. Por último, en la octava sección, se resumen las conclusiones del trabajo.

\section{Obtención del modelo}

De acuerdo a Böhm \& Kaas (2000), la acumulación de capital está dada por: 


$$
k_{t+1}=\frac{1}{1+n}\left[(1-\delta) k_{t}+s_{w}\left(f\left(k_{t}\right)-k_{t} f^{\prime}\left(k_{t}\right)\right)+s_{r} f^{\prime}\left(k_{t}\right)\right]
$$

donde $\delta \epsilon(0,1)$ es la tasa de depreciación del stock de capital, $n$ es la tasa de crecimiento de la fuerza de trabajo, $s_{w} \in(0,1)$ y $s_{r} \in(0,1)$ son las tasas de ahorro constante de los trabajadores y de los accionistas respectivamente, asumiendo $s_{w} \neq s_{r}$.

Consideraremos ahora la ecuación de producción y la elección del mapa que describa la fuerza de trabajo:

i. La función de producción que vincula el stock de capital con el producto por trabajador es de tipo CES, introducida en 1961 por Arrow et al. (1961):

$$
y=f(k)=\left(1+k^{\rho}\right)^{\frac{1}{\rho}}
$$

donde $\rho<1, \rho \neq 0$.

La función de producción CES generaliza tres funciones de producción bien definidas según el valor que le otorguemos a la elasticidad de substitución entre los factores capital y trabajo. En la Figura 1 se pueden apreciar las diferencias y similitudes de las funciones de producción resultantes

Figura 1: Funciones de producción Lineal, Cobb-Douglas, CES con $\rho=-2$ y Leontief.

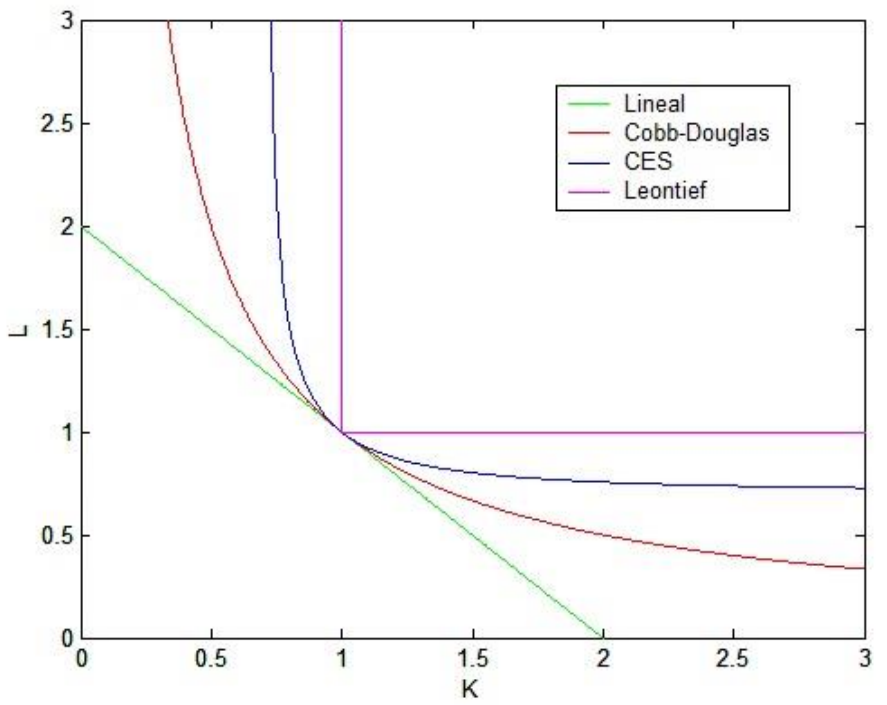

Fuente: elaboración propia.

ii. La tasa de crecimiento para la fuerza de trabajo debe ser no constante. En Brianzoni et al. (2007a), la tasa de crecimiento es modelada por la ecuación de Beverton-Holt: 


$$
n_{t+1}=\frac{r h}{h+(r-1) n_{t}} n_{t}
$$

donde $h>0$ es la capacidad de acarreo (por ejemplo, la disponibilidad de recursos) y $r>0$ es la tasa de crecimiento intrínseca. Por otra parte, en Brianzoni et al. (2007b), la misma es descripta por el bien conocido mapa logístico:

$$
n_{t+1}=\mu n_{t}\left(1-n_{t}\right)
$$

con $\mu \in(1,4)$ para el cual la dinámica es no trivial pero invariante en el intervalo $[0,1]$.

En este trabajo se propone modelar la tasa de crecimiento de la fuerza de trabajo mediante el mapa de Richards (Radwan, 2013; Richards, 1959) en tiempo discreto:

$$
n_{t+1}=r n_{t}\left(1-n_{t}^{\gamma}\right)
$$

donde $y>0$ es un parámetro que permite lograr mayor flexibilidad en la ubicación del punto de inflexión de la curva, con la consecuente aparición de nuevas fluctuaciones. Las distintas variantes que presenta el mapa de Richards en función de sus parámetros pueden ser apreciadas en la Figura 2.

Figura 2: Mapa de Richards con $r=2$ y $\gamma=0,5,2$ y 6 .

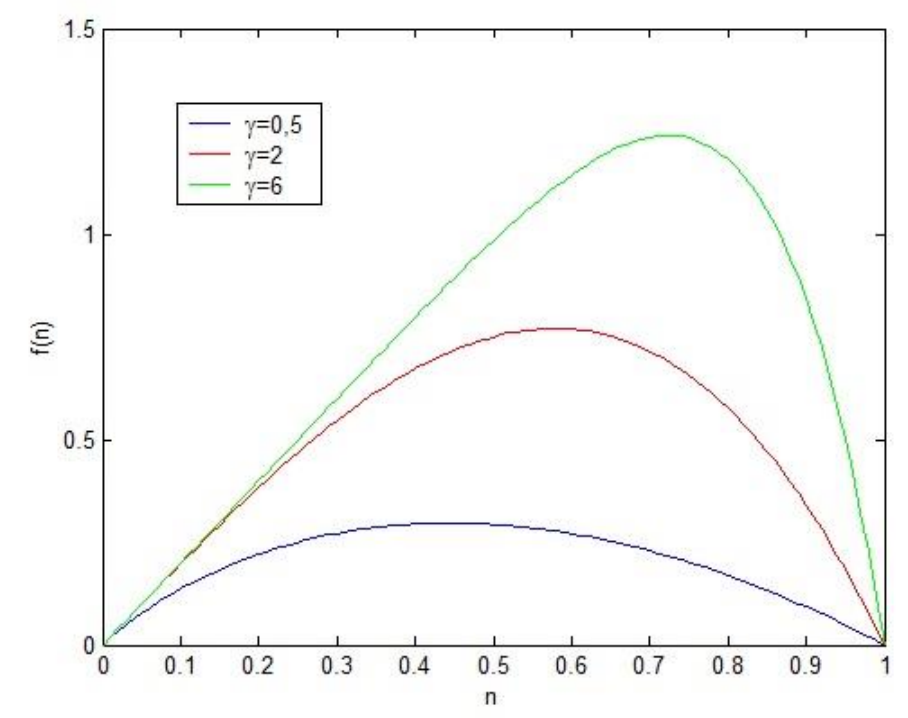

Fuente: elaboración propia.

Relacionando (1), (2) y (3), resulta: 


$$
\left\{\begin{array}{l}
n_{t+1}=r n_{t}\left(1-n_{t}^{\gamma}\right) \\
k_{t+1}=\frac{1}{1+n_{t}}\left[(1-\delta) k_{t}+\left(k_{t}^{\rho}+1\right) \frac{1-\rho}{\rho}\left(s_{w}+s_{r} k_{t} \rho\right)\right]
\end{array}\right.
$$

que es un sistema dinámico discreto determinado por un mapa triangular $T$ en el plano:

$$
T: \square_{+}^{2} \rightarrow \square_{+}^{2}, T\left(\begin{array}{l}
n \\
k
\end{array}\right)=\left(\begin{array}{l}
f(n) \\
g(n, k)
\end{array}\right)
$$

$\operatorname{con} f(n)=r n\left(1-n^{\gamma}\right)$ y $g(n, k)=\frac{1}{1+n}\left[(1-\delta) k+\left(k^{\rho}+1\right) \frac{1-\rho}{\rho}\left(s_{w}+s_{r} k^{\rho}\right)\right]$.

\section{Estabilidad de puntos fijos}

Los puntos fijos del mapa $T$ son las soluciones del sistema algebraico $T(n, k)=(n, k)$. De la primera ecuación se deduce que los puntos fijos pertenecen a las rectas $n=0$ y $n=n^{*}=\left(\frac{r-1}{r}\right)^{\frac{1}{\gamma}}$

De la segunda ecuación tenemos que la segunda coordenada $k$ de los puntos fijos de $T$ son los puntos fijos de los mapas unidimensionales $g_{0}=(k):=g(0, k)$ y $g_{n^{*}}=(k):=g\left(n^{*}, k\right)$. Dependiendo de los parámetros del modelo, por utilizar la función de producción CES y la ecuación de Richards, estos modelos pueden tener múltiples equilibrios. La estructura triangular de $T$ hace más sencillo el análisis debido a que la matriz jacobiana de $T$ tiene autovalores reales ubicados en su diagonal principal dados por

$$
\lambda_{1}(n)=r-r(\gamma+1) n^{\gamma} \text { y } \lambda_{2}(n, k)=\frac{\partial g(n, k)}{\partial k} .
$$

Por resultar $\lambda_{1}(0)>1$ los puntos fijos que pertenecen a la recta $n=0$ pueden ser saddles o nodos inestables. Por otro lado $\left|\lambda_{1}\left(n^{*}\right)\right|<1$ se verifica si y sólo si $r \in(1,(2 / Y)+1)$, por lo que la recta $n=n^{*}$ atrae las trayectorias con las condiciones iniciales (no, ko) si $n_{0} \in$ $(0,1)$. Los parámetros $\rho, \delta, s_{r}$ y $s_{w}$ inciden de forma directa tanto en el comportamiento de $g_{n *}(k)$, la cual puede resultar monótona o bien bimodal en $k$, como en el valor de $\left|\lambda_{2}(n *, k)\right|$. Mediante un estudio detallado se obtienen los siguientes resultados: 
i. Supongamos $\rho \in(0,1)$. Si $s_{r}<\left(\frac{r-1}{r}\right)^{\frac{1}{\gamma}}+\delta$ entonces $T$ tiene un punto fijo con $n=n^{*} . g(n *, k)$ resulta creciente y cóncava por lo que $\left|\lambda_{2}\right|<1$ y dicho punto fijo es asintóticamente estable. En caso contrario $T$ no posee puntos fijos con $n \neq 0$.

ii. Supongamos que $\rho<0$. Se distinguen los siguientes casos:

a) Asumamos que $s_{r}<s_{w}$ y $\rho<\frac{s_{r}}{s_{r}-s_{w}}$. Si $s_{r}>\left(\frac{r-1}{r}\right)^{\frac{1}{\gamma}}+\delta$ entonces $T$ posee un punto fijo con $n=n^{*}$ que es asintóticamente estable. En caso contrario $T$ no posee puntos fijos con $n \neq 0$.

b) Asumamos que $s_{r}<s_{w}$ y $\rho<\frac{s_{r}}{s_{r}-s_{w}}$. Entonces $T$ posee dos puntos fijos con $n=n *$, uno es de tipo saddle y el otro asintóticamente estable.

c) Asumamos que $s_{r}<s_{w}$. Si $s_{r} \leq\left(\frac{r-1}{r}\right)^{\frac{1}{\gamma}}+\delta, T$ no posee puntos fijos con $n \neq 0$. Si $s_{r}>\left(\frac{r-1}{r}\right)^{\frac{1}{\gamma}}+\delta$ y además $\rho>\frac{-s_{w}}{s_{r}-s_{w}}$, entonces $T$ posee un punto fijo con $n=n *$ que es asintóticamente estable.

Si se cumple $\left\{\rho<\frac{-s_{w}}{s_{r}-s_{w}}\right\} \wedge\left\{\varphi\left(\rho, s_{r}, s_{w}\right)>\delta-\left(\frac{r-1}{r}\right)^{\frac{1}{\gamma}}-2\right\}$, donde
$\varphi\left(\rho, s_{r}, s_{w}\right)=\rho\left(s_{r}-s_{w}\right)\left[\frac{\rho\left(s_{r}-s_{w}\right)+s_{w}}{(2 \rho-1)\left(s_{r}-s_{w}\right)}\right]^{\frac{2 \rho-1}{\rho}}$, entonces $T$ posee un punto fijo con $n=n *$ que es asintóticamente estable.

\section{Ciclos y dinámicas complejas}

Para indagar en la aparición de otros comportamientos dinámicos, consideraremos valores de los parámetros no contemplados en el esquema anterior. 
Si se cumplen las condiciones del ítem (ii)-c) pero $\varphi\left(\rho, s_{r}, s_{w}\right)<\delta-1$, podemos demostrar que el ingreso del capital dado por $s_{r} f_{k}^{\prime}\left(k_{t}\right) k_{t}$ no es monótono y $g_{n *}(k)$ resulta bimodal, razón por la cual aparecen ciclos o caos en la dinámica de (4). Ejemplos sobre la aparición de ciclos se muestran en la Figura 3, mediante el recurso gráfico conocido como cobweb.

Es más, para $r=1,1$ y $\rho \in(-70,-10)$, se detecta una ruta al caos por duplicación de período que se ilustra en el diagrama de bifurcación de la Figura 4(a).

Además, si $r>(2 / Y)+1$, la dinámica de (3) desarrolla una ruta al caos por duplicación de período como puede apreciarse en el diagrama de bifurcación de la Figura 4(b), con la consiguiente aparición de este comportamiento en la dinámica de (4).

Figura 3: (a) Ciclo 3-periódico para $\delta=0,4, \rho=-50, r=1,1, \quad \gamma=1,1, s_{r}=0,8$ y $s_{w}=0,1$. (b) Ciclo de mayor período para los mismos valores de parámetros que en (a) pero con $s_{r}=0,9$.
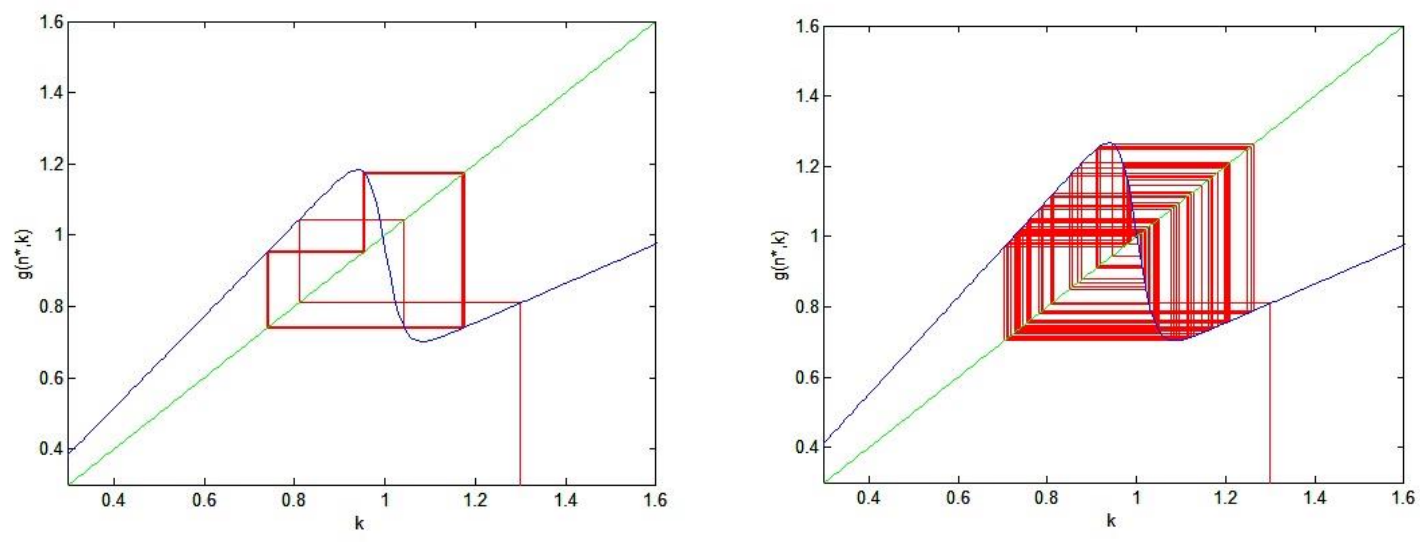

Fuente: elaboración propia.

\section{Simulaciones numéricas}

Realizaremos algunas simulaciones numéricas para entender mejor la dinámica global de nuestro modelo. Fundamentalmente describiremos las bifurcaciones que incrementan la complejidad de la dinámica asintótica del sistema, teniendo en cuenta lo desarrollado en la sección anterior.

De todos los parámetros involucrados, se analizarán en los correspondientes diagramas de fases las variaciones debidas a $\rho$ y a $r$, recordando que estos parámetros son los que juegan un rol importante para la aparición de dinámicas complejas. 
Figura 4: (a) Diagrama de bifurcación de $\rho$ para $\delta=0,4, r=1,1, \gamma=1,1, s_{r}=0,6$ y $s_{w}=0,1$. (b) Diagrama de bifurcación de $r$ para $\delta=0,4, \rho=-50, \gamma=1,1, s_{r}=0,9$ y $s_{w}=0,1$.
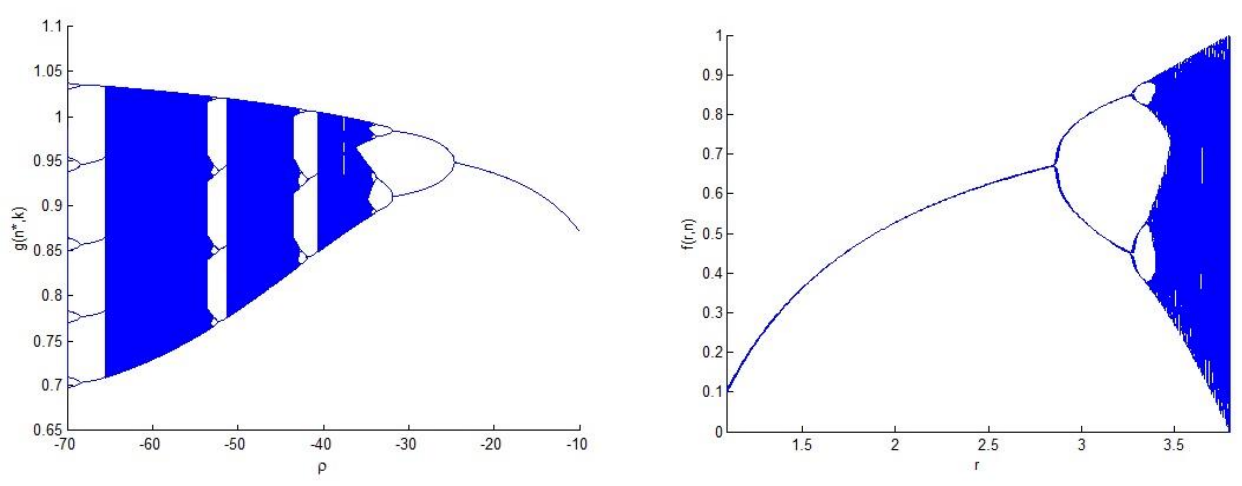

Fuente: elaboración propia.

En la Figura 5 podemos apreciar la influencia del parámetro $r$. Vemos que en la Figura 5(a) el diagrama de fases es cíclico 4-periódico, pero a medida que $r$ aumenta, una secuencia de duplicación de período da lugar a ciclos de período $2^{k}$ y luego a un diagrama de fases cíclico caótico 4-periódico, evidenciado en la Figura 5(b). En esta situación el comportamiento de largo plazo del sistema está caracterizado por ciclos 4-periódicos, aunque en cada período no podemos predecir el estado exacto.

Figura 5: (a) Diagrama de fases cíclico de período 4 con $\rho=-50, \delta=0,01, r=3,2$, $\gamma=1,1, s_{r}=0,9$ y $s_{w}=0,1$. (b) Diagrama de fases cíclico caótico de período 4 con $\rho=-50, \delta=0,01, r=3,5, \gamma=1,1, s_{r}=0,9$ y $s_{w}=0,1$.
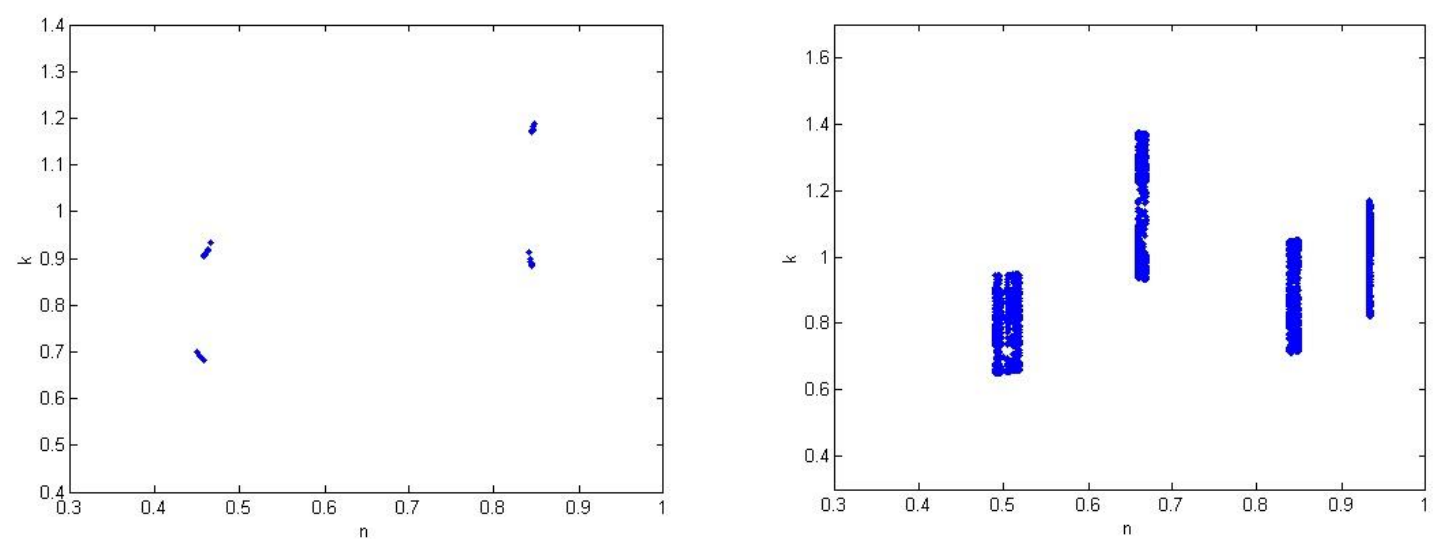

Fuente: elaboración propia.

En la Figura 6 podemos apreciar la influencia del parámetro $\rho$. En la Figura 6(a) vemos 
que, con valores elevados de $r$, el diagrama de fases muestra un comportamiento caótico aún para valores altos de $\rho$, y a medida que $\rho$ disminuye el diagrama de fases mostrado en la Figura 6(b) evidencia un comportamiento caótico más complejo aún. En esta situación el comportamiento de largo plazo del sistema se caracteriza por haber reemplazado el comportamiento cíclico por una evolución totalmente errática que cubre una gran parte del diagrama de fases del sistema dinámico.

Figura 6: (a) Diagrama de fases caótico con $\rho=-10, \delta=0,01, r=3,7, \gamma=1,1,{ }_{r}=0,9$ y $s_{w}=0,1$. (b) Diagrama de fases caótico con $\rho=-50, \delta=0,01, r=3,7, \gamma=1,1$, $s_{r}=0,9$ y $s_{w}=0,1$.
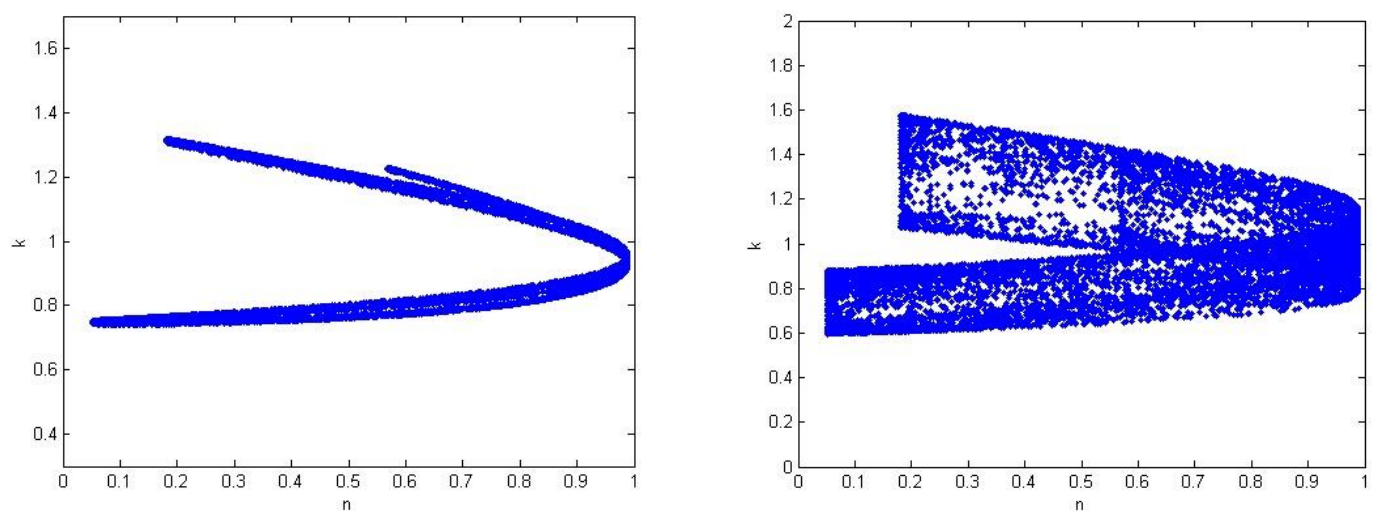

Fuente: elaboración propia.

\section{Cuantificación del caos mediante exponentes de Lyapunov}

A partir de ahora introduciremos una forma alternativa de poner en evidencia la existencia del caos a través de un número que lo cuantifique, partiendo del hecho que una órbita caótica es aquella que continúa experimentando indefinidamente un comportamiento inestable tal como una órbita cualquiera lo haría en las cercanías de una fuente, pero donde la misma no es ni periódica ni tampoco un punto fijo.

Si $f$ es un mapa y $x_{1}$ un punto fijo del mismo, y además se cumple que $f^{\prime}\left(x_{1}\right)=a>1$, entonces la órbita de cada punto $x$ cercano a $x_{1}$ se separará de este a una tasa de aproximadamente $a$ en cada iteración. Por lo tanto, la distancia entre $f^{n}(x)$ y $f^{n}\left(x_{1}\right)=x_{1}$, irá aumentando aproximadamente $a$ veces por cada iteración.

El concepto de número de Lyapunov es el apropiado para cuantificar esta tasa promedio de separación de los puntos $x$ que estén cercanos a $x_{1}$. El exponente de Lyapunov será tan sólo el logaritmo natural del número de Lyapunov. Siguiendo con el ejemplo dado, si el número de Lyapunov fuese $C$, para cada punto periódico $x_{1}$ de período $k$, tendríamos: 
$\left|\left(f^{k}\right)^{\prime}\left(x_{1}\right)\right|=\left|f^{\prime}\left(x_{1}\right)\right|\left|f^{\prime}\left(x_{1}\right)\right| \cdots\left|f^{\prime}\left(x_{1}\right)\right|=C^{k}$

Supongamos que $f$ sea un mapa suave en la recta real. Entonces el número de Lyapunov $L\left(x_{1}\right)$ de la órbita $\left\{x_{1}, x_{2}, x_{3}, \ldots, x_{n}\right\}$ se define como:

$L\left(x_{1}\right)=\lim _{n \rightarrow \infty}\left(\left|f^{\prime}\left(x_{1}\right)\right|\left|f^{\prime}\left(x_{2}\right)\right| \cdots\left|f^{\prime}\left(x_{n}\right)\right|\right)^{1 / n}$

si ese límite existe. El exponente de Lyapunov $h\left(x_{1}\right)$ se define como:

$h\left(x_{1}\right)=\lim _{n \rightarrow \infty}(1 / n)\left[\ln \left|f^{\prime}\left(x_{1}\right)\right|+\ln \left|f^{\prime}\left(x_{2}\right)\right|+\cdots+\ln \left|f^{\prime}\left(x_{n}\right)\right|\right]$

si ese límite existe. Debemos notar que $h$ existe sí y sólo sí $L$ existe, y $\ln L=h$.

Supongamos que $f$ sea un mapa suave en la recta real y además $\left\{x_{1}, x_{2}, \ldots\right\}$ una órbita acotada de $f$. Entonces la órbita es considerada caótica si:

i) $\left\{x_{1}, x_{2}, \ldots\right\}$ no es asintóticamente periódica.

ii) El exponente de Lyapunov $h\left(x_{1}\right)$ es mayor que cero.

Los conceptos estudiados anteriormente pueden ser generalizados a mapas en donde $m \geq 1$. La idea de medir tasas de separación entre puntos cercanos en el caso unidimensional, debe ser ampliada dado que en dimensiones mayores el comportamiento local puede depender también de la dirección, y entonces tendremos un número de Lyapunov por cada dimensión del mapa en cuestión.

Supongamos que $f$ sea un mapa de, $m \geq 1, y$ asumamos que $\left\{v_{0}, v 1, v_{2}, \ldots\right\}$ sea una órbita acotada de $f$. La órbita es considerada caótica si:

i) $\left\{v_{0}, v 1, v_{2}, \ldots\right\}$ no es asintóticamente periódica.

ii) Ningún número de Lyapunov es exactamente uno.

iii) $L_{1}\left(v_{0}\right)>1$.

En términos de los exponentes de Lyapunov, la parte (iii) de la definición anterior es equivalente a afirmar que $h_{1}\left(v_{0}\right)>0$.

A continuación, se representarán los exponentes de Lyapunov correspondientes a nuestro modelo, es decir $h_{1}$ y $h_{2}$ para distintos valores de los parámetros $r$ y $\rho$.

En la Figura 7 podemos apreciar como varían los exponentes de Lyapunov $h_{1}$ y $h_{2}$ cuando $r$ varía desde 1 hasta 3,7; aquellos que pueden ser calculados y que se sitúan por arriba de cero son los que indican la presencia de órbitas caóticas. 
Figura 7: Exponentes de Lyapunov $h_{1}$ y $h_{2}$ en función de $r$ con $\rho=-2, \delta=0,2$,

$$
s_{r}=0,9, s_{w}=0,1 \text { y } \gamma=1,1 \text {. }
$$
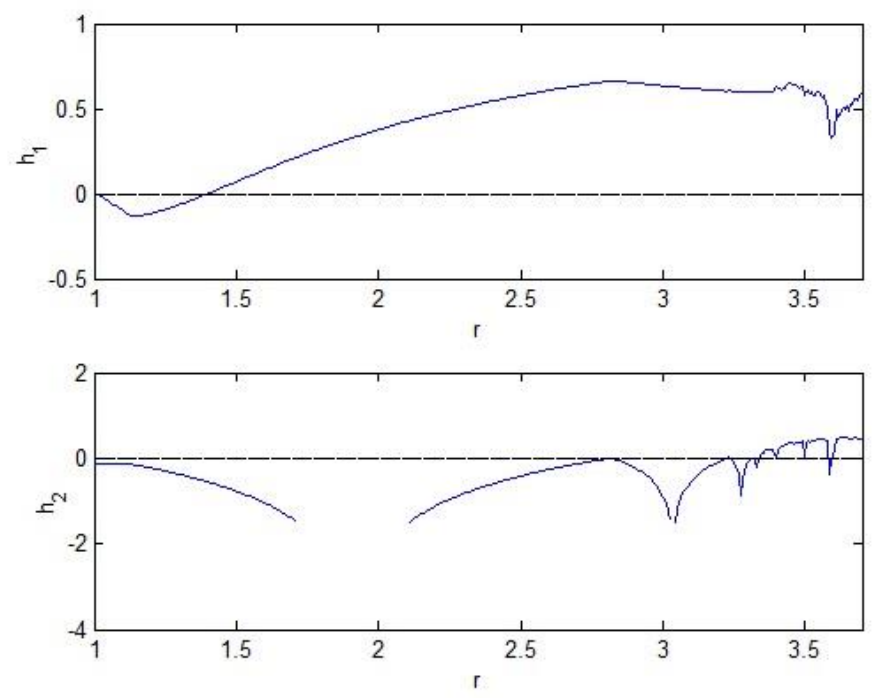

Fuente: elaboración propia.

En la Figura 8 podemos apreciar como varían los exponentes de Lyapunov $h_{1}$ y $h_{2}$ cuando $\rho$ varía desde $-2,2$ hasta $-0,01$; aquellos que se sitúan por arriba de cero son los que indican la presencia de órbitas caóticas:

Figura 8: Exponentes de Lyapunov $h_{1}$ y $h_{2}$ en función de $\rho$ con $\delta=0,2, s_{r}=0,9$,

$$
s_{w}=0,1, r=2,5 \text { y } \gamma=1,1 \text {. }
$$
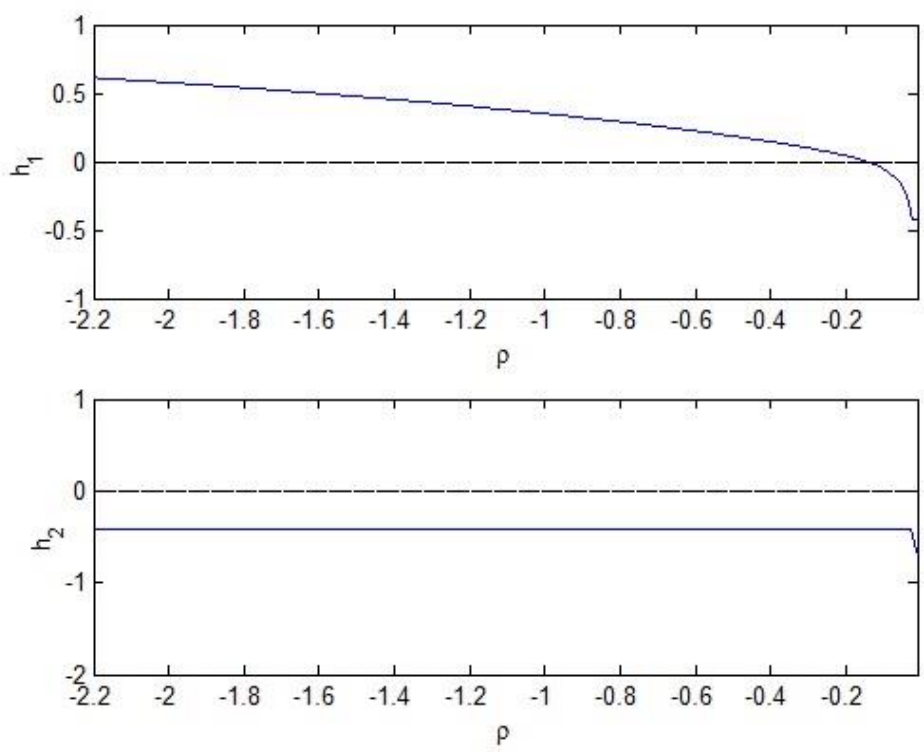

Fuente: elaboración propia. 


\section{Interpretación del modelo}

El parámetro $\rho$ de la función de producción CES está relacionado con la elasticidad de substitución entre los factores capital y trabajo $\sigma$, dada por $\sigma=1 / 1-\rho$. La consideración económica resultante es que, si $\rho$ resulta mayor que cero, la elasticidad de substitución es elevada; si además el parámetro $r$ de la ecuación de Richards es bajo, no se manifestarán dinámicas complicadas. Este caso correspondería a una economía no desarrollada, cuya población aún está lejos de su capacidad de acarreo del entorno y que podría exhibir un crecimiento económico con menor volatilidad.

Si la producción de una economía presenta una escasa elasticidad de substitución entre los factores productivos, es decir cuando resulta $\rho<0$ como en el caso de una economía desarrollada con alto stock de capital de contenido tecnológico y además alto capital humano, la diferencia entre las tasas de ahorro de los accionistas y la de los trabajadores juega un papel fundamental en cuanto a la aparición de dinámicas complejas. En esa situación, la monotonicidad del Ingreso de capital no se verifica si $\rho$ es lo suficientemente bajo $y$, además $s_{r}>s_{w}$, con la consiguiente aparición de dinámicas complejas y eventualmente caos.

\section{Conclusiones}

En este trabajo se ha investigado la estabilidad local y las propiedades globales de un modelo de crecimiento de Solow en tiempo discreto con tasas de ahorro diferencial constante y crecimiento poblacional no constante. Las variantes novedosas del mismo han consistido en utilizar la función de producción CES y la ecuación de Richards para modelizar la fuerza de trabajo, con una tasa de crecimiento poblacional variable y la posibilidad de poder elegir con mayor flexibilidad el punto de inflexión de la misma dada por el parámetro.

El análisis de los puntos fijos concluyó la existencia de equilibrios múltiples según determinadas condiciones de valores de los parámetros del sistema. Luego se analizaron otros valores de los parámetros no contemplados en el análisis preliminar mediante el recurso gráfico cobweb, diagramas de bifurcación y diagramas de fases, especialmente para poner en evidencia las dinámicas complejas a las que puede arribar el presente modelo. Posteriormente el análisis de dichas fluctuaciones se complementó con la obtención de los exponentes de Lyapunov para poder cuantificar la existencia de caos.

Los resultados del análisis muestran que el modelo estudiado puede exhibir fluctuaciones, dinámicas complejas, ciclos de múltiples períodos, y también comportamiento caótico. Estas características se manifiestan a medida que el parámetro de la ecuación de Richards aumenta (lo cual produce un aumento en la fluctuación de la tasa de crecimiento poblacional) o también si el parámetro disminuye (lo cual también impacta en la elasticidad de substitución de la función de producción).

De acuerdo a esto, ha quedado en evidencia el rol central que juega la elasticidad de substitución en relación a la propiedad de monotonicidad del ingreso de capital, dado 
que las dinámicas complejas emergen y propagan sus fluctuaciones al fallar esta condición.

En el caso de economías no desarrolladas, que verifican alta elasticidad de substitución entre los factores capital y trabajo, y manifiestan una baja tasa de crecimiento poblacional, no es factible la aparición de fluctuaciones. Dichas dinámicas son propensas a manifestarse cuando la elasticidad de substitución es baja, tal es el caso de una economía desarrollada con alto contenido de capital de índole tecnológico y elevado capital humano, verificando estas economías también los valores de tasas de ahorro diferenciales propicias al surgimiento de este fenómeno.

En este trabajo se han presentado las primeras herramientas analíticas y numéricas que permiten inferir la riqueza de esta dinámica según se varíen los valores de los parámetros del sistema. A futuro, se planea profundizar en las mismas con el objetivo de conseguir una descripción más detallada de esta dinámica. 


\section{Referencias}

Arrow, K. J., Chenery, H. B., Minhas, S., \& Solow, R. M. (1961). Capital-labor substitution and economic efficiency. The Review of Economics and Statistics, 43(3), 225-250.

Benhabib, J., \& Nishimura, K. (1985). Competitive equilibrium cycles. Journal of Economic Theory, 35(2), 284-306.

Böhm, V., \& Kaas, L. (2000). Differential savings, factor shares and endogenous growth cycles. Journal of Economic Dynamics \& Control, 24(5-7), 965-980.

Boldrin, M., \& L. Montrucchio, L. (1986). On the indeterminacy of capital accumulation paths. Journal of Economic Theory, 40(1), 26-39.

Brianzoni, S., Mammana, C., \& Michetti, E. (2007a). Complex dynamics in the neoclassical growth model with differential savings and non-constant labor force growth. Studies in Nonlinear Dynamics \& Econometrics, 11(3), 1-19.

Brianzoni, S., Mammana, C., \& Michetti, E. (2007b). Nonlinear dynamics in a businesscycle model with logistic population growth. Chaos, Solitons and Fractals, 40(2), 717730.

Chiang, A. C. (1973). A simple generalisation of the Kaldor-Pasinetti theory of profit rate and income distribution. Economica, 40(159), 311-313.

Kaldor, N. (1955). Alternative theories of distribution. Review of Economic Studies, 23(2), 83-100.

Radwan, A. G. (2013). On some generalized discrete logistic maps. Journal of Advanced Research, 4(2), 163-171.

Richards, F. J. (1959). A flexible growth function for empirical use. Journal of Experimental Botany, 10(2), 290-300.

Solow, R. M. (1956). A contribution to the theory of economic growth. The Quarterly Journal of Economics, 70(1), 65-94. 